\title{
Hydroxytyrosol inhibits the inflammatory response of osteoarthritis chondrocytes via SIRT6-mediated autophagy
}

\author{
LI-QIANG ZHI ${ }^{*}$, SHU-XIN YAO ${ }^{1 *}$, HONG-LIANG LIU ${ }^{2},{\text { MENG } \text { LI }^{3}, \text { NING DUAN }^{4} \text { and JIAN-BING MA }}^{1}$ \\ Departments of ${ }^{1}$ Joint Surgery and ${ }^{2}$ Orthopaedic Trauma, Hong-Hui Hospital, Xi'an Jiaotong University, Xi'an, Shanxi 710054; \\ ${ }^{3}$ Department of Orthopedics, The First Affiliated Hospital of Xi'an Jiaotong University, Xi'an, Shaanxi 710061; \\ ${ }^{4}$ Department of Traumatic Osteopathic, Hong-Hui Hospital, Xi'an Jiaotong University, Xi'an, Shaanxi 710054, P.R. China
}

Received December 14, 2016; Accepted November 24, 2017

DOI: $10.3892 / \mathrm{mmr} .2017 .8353$

\begin{abstract}
Osteoarthritis (OA) is a common degenerative joint disease. Inflammation may exaggerate the catabolism and degeneration in the pathogenesis of OA. Hydroxytyrosol (HT) has been used in the management of inflammatory diseases. In addition, reports have revealed that autophagy was a therapeutic target of diseases caused by inflammation. Sirtuin 6 (SIRT6) has also been demonstrated to prevent OA development by reducing both the inflammatory response and chondrocyte senescence. However, the roles of SIRT6 and autophagy in cartilage and its underlying anti-inflammatory mechanism are unknown. Therefore, the present study aimed to determine the effects of HT on autophagy and inflammation in chondrocytes, and clarify whether HT regulates the inflammatory response through SIRT6-mediated autophagy. The expression of protein and mRNA were determined by western blot analysis and reverse transcription-quantitative polymerase chain reaction. The production of cytokines was detected by ELISA. It was demonstrated that HT inhibited the levels of interleukin (IL)- $1 \beta$ and IL- 6 in tumor necrosis factor (TNF)- $\alpha$-stimulated chondrocytes in a concentration-dependent manner. In addition, HT promoted cell autophagy and increased the mRNA and protein expression levels of SIRT6 in chondrocytes stimulated with TNF- $\alpha$. Autophagy inhibitor 3-methyladenine or knockdown of SIRT6 decreased the inhibitory effects of HT on the inflammatory response in chondrocytes. In addition, knockdown of SIRT6 attenuated the expression of microtubule-associated protein 1A/1B-light chain 3 and Beclin1 in chondrocytes. Overall, these findings suggested that HT inhibits the inflammatory response of chondrocytes through
\end{abstract}

Correspondence to: Professor Jian-Bing Ma, Department of Joint Surgery, Hong-Hui Hospital, Xi'an Jiaotong University, 76 Nanguo Road, Xi'an, Shaanxi 710054, P.R. China

E-mail: vitosun@sina.com

${ }^{*}$ Contributed equally

Key words: hydroxytyrosol, chondrocytes, sirtuin 6, autophagy, inflammation
SIRT6-mediated autophagy. The present study provided a new drug target for the clinical treatment of inflammatory diseases.

\section{Introduction}

Osteoarthritis (OA) is a common degenerative joint disease which is predominantly characterized by irreversible loss of the cartilage and inflammation of synovium (1). Chondrocytes are the only resident cells in articular cartilage and their function is an important factor in determining the pathogenesis of OA (2). Notably, previous studies have demonstrated that inflammation is induced by pro-inflammatory cytokines, which also serve a critical role in the development and progression of OA (3-5). It has also been indicated that inflammatory responses lead to an increase in apoptosis and dysfunction of chondrocytes, and as a result, the catabolism, degeneration and pathogenesis associated with $\mathrm{OA}$ are exaggerated. Further studies have verified that the inflammatory response is induced by interleukin (IL)-1 $\beta$ (3), a pro-inflammatory and pro-catabolic cytokine, which leads to upregulation of nitric oxide and prostaglandin E2 by producing more matrix metalloproteinases and less extracellular matrix. Therefore, the present study aimed to identify a promising compound that may inhibit inflammatory mediator production, and possesses the ability to improve the viability and function of chondrocytes, therefore impeding the progress of OA (5).

Autophagy, an evolutionarily conserved self-protective mechanism (6), is highly efficient in protecting physiological processes by removing protein aggregates or unnecessary cellular components. Furthermore, it has been demonstrated that autophagy is involved in inflammatory reactions and downregulation of autophagy in macrophages leads to inflammation (7). In addition, a previous study reported that resveratrol inhibits endothelial inflammation via autophagy induction (8). However, the regulatory mechanisms for autophagy in inflammation are also complex and remain to be fully elucidated. Sirtuin (SIRT)6, an $\mathrm{NAD}^{+}$-dependent deacetylase, belongs to class III histone/protein deacetylases and is a member of the silent information regulator 2 family $(9,10)$. In the Sirtuin family, SIRT6 and SIRT1 are predominantly localized in the nucleus and share functional similarities. It has been reported that SIRT1 mediates human and mice chondrocyte survival in the context of arthritis. It has 
also been indicated that knockdown of SIRT1 in chondrocytes lessens cartilage-specific gene expression and accelerates hypertrophy and cartilage matrix loss. Additionally, a previous study revealed that fenofibrate prevented high glucose-induced inflammatory responses in cardiac myoblasts by upregulating SIRT1-mediated autophagy (11). Previously, SIRT6 has gained considerable research attention owing to the newly discovered roles it serves in autophagy (12) and osteoarthritis development. There is research that suggests that overexpression of SIRT6 suppresses inflammatory responses in tumor necrosis factor (TNF)- $\alpha$ induced synoviocytes and bone destruction (13). SIRT6 overexpression induces autophagy via attenuation of insulin-like growth factor-protein kinase B-mechanistic target of rapamycin signaling. Conversely, SIRT6 inhibition suppresses autophagy. Furthermore, SIRT6 has been demonstrated to prevent OA development by reducing the inflammatory response and chondrocyte senescence (14). However, the roles of SIRT6 in cartilage and its anti-inflammatory mechanism remain unknown, and require further investigation. Therefore, it was hypothesized that the activation of SIRT6 serves a role in the regulation of autophagy, with subsequent inhibitory effects on inflammation in chondrocytes and OA progression.

Hydroxytyrosol (HT) is a phenol molecule derived from olive leaves and olive oil and is part of the traditional Mediterranean diet $(15,16)$. Previous studies have indicated that consumption of HT exhibits certain health benefits on various physiological functions due to its anti-oxidative and anti-inflammatory pharmacological activities (17). Furthermore, it has been demonstrated that HT inhibits the inflammatory response in vascular endothelial cells, macrophages and monocytes (18). It has also been demonstrated that HT increases the autophagy of chondrocytes and preosteoblast cells and protects them from cell death and oxidative damage $(19,20)$, respectively. In addition, the study revealed that overexpression of SIRT6 could suppress inflammation in a variety of cells $(19,20)$. However, the roles of SIRT6 and autophagy in the anti-inflammatory process of HT in chondrocytes remains unknown. Therefore, elucidating the potential underlying anti-inflammatory mechanisms of HT and the involvement of SIRT6 and autophagy may be valuable in treating inflammation-associated diseases including OA.

\section{Materials and methods}

Chemical reagents and antibodies. HT (purity >98\%) was purchased from Xi'an App-Chem Bio (Tech) Co., Ltd. (Shaanxi Sheng, China). Tumor necrosis factor- $\alpha$ (TNF- $\alpha$ ) was an inflammatory induction factor obtained from PeproTech, Inc. (Rocky Hill, NJ, USA). The antibodies used in this experiment are included SIRT6, LC3, Beclin1, MCP-1, $\beta$-actin and horse radish peroxidase conjugated anti-rabbit secondary antibody. SIRT6 antibody (cat. no. 12486, Cell Signaling Technology, Inc., Danvers, MA, USA) was used as a primary antibody. Polyclonal anti-microtubule-associated protein 1A/1B-light chain 3 (LC3; cat. no. 12741) and anti-Beclin1 antibodies (cat. no. 3495) were provided by Cell Signaling Technology, Inc. Antibodies against monocyte chemoattractant protein 1 (MCP-1; cat. no. NBP2-22115) were purchased from Novus Biologicals, LLC, Littleton, CO, USA. Antibodies against $\beta$-actin (cat. no. 4970) were purchased from Cell Signaling Technology, Inc. and horse radish peroxidase conjugated anti-rabbit secondary antibody was provided by Santa Cruz Biotechnology, Inc. (Santa Cruz, CA, USA). Lipofectamine ${ }^{\circledR}$ 2000 was obtained from Invitrogen (Thermo Fisher Scientific, Inc., Waltham, MA, USA). RNAiso Plus, PrimeScript RT Master mix and SYBR Premix Ex Taq II were purchased from Takara Bio, Inc. (Otsu, Japan). A rat IL-1 $\beta$ ELISA kit was obtained from Cusabio Biotech Co., Ltd (cat. no. KET9001). (Newark, DE, USA). The mRNA primers were synthesized by Generay Biotech Co., Ltd. (Shanghai, China). 3-methyladenine (3-MA) was purchased from Sigma-Aldrich; Merck KGaA (Darmstadt, Germany). Rat IL-1 $\beta$ and IL-6 enzyme-linked immunosorbent assay (ELISA) kit was obtained from Cusabio Biotech Co., Ltd. (cat. no. KET9001). All other chemicals were of high purity and were obtained from commercial sources.

Cell culture. The approval for the use of the animals in this study was granted by the Animal Ethics Committee of Xi'an Jiaotong University (Xi'an, China). Adult male Sprague-Dawley rats were provided by the Experimental Animal Center of Xi'an Jiaotong University (Animal certificate no. SYXK (shan) 2014-003). A total of 2 Male Sprague-Dawley rats (200-300 g) were euthanized as previously described immediately upon receipt (21). The knee cartilages were separated under microscopy and digested with $0.25 \%$ trypsin-EDTA for $30 \mathrm{~min}$ and collagenase II for $4 \mathrm{~h}$ at $37^{\circ} \mathrm{C}$, and then the chondrocytes were filtered to produce a single cell suspension. Chondrocytes were cultured in high-glucose (4.5 g/l) Dulbecco's modified Eagle's medium (DMEM; Hyclone; GE Healthcare Life Sciences, Logan, UT, USA) with antibiotics (1\% penicillin and streptomycin) and $10 \%$ fetal bovine serum (Hyclone; GE Healthcare Life Sciences) in an incubator containing $5 \% \mathrm{CO}_{2}$ at $37^{\circ} \mathrm{C}$, Passages between 2 and 4 were used for the experiments.

Cell viability assay. A Cell Counting kit (CCK)-8 assay (Roche Applied Science, Penzberg, Germany) was performed to determine cell viability. Briefly, chondrocytes were seeded at a density of $2 \times 10^{3}$ cells well in 96-well microplates. Following incubation for $24 \mathrm{~h}$, HT was added to the culture medium at different concentrations $(0,12.5,25,50,100,200$ and $400 \mu \mathrm{M})$ and then treated with TNF- $\alpha(5 \mathrm{ng} / \mathrm{ml})$ for $24 \mathrm{~h}$ at $37^{\circ} \mathrm{C}$. The cells were then cultured with fresh media for an additional $48 \mathrm{~h}$ at $37^{\circ} \mathrm{C}$. CCK-8 solution was added (10 $\mu \mathrm{l}$ well), and the cells were further incubated for $3 \mathrm{~h}$ at $37^{\circ} \mathrm{C}$ in a $5 \%$ (v $\left./ \mathrm{v}\right) \mathrm{CO}_{2}$ atmosphere. The optical density was measured using a microplate reader (Thermo Fisher Scientific, Inc.) at a wavelength of $450 \mathrm{~nm}$, with a background control of media and CKK-8 solution as a blank.

ELISA. Following different treatment conditions, cell culture supernatants were collected. The sample was centrifuged at a rate of $200 \times \mathrm{g}$ for $10 \mathrm{~min}$ at $4^{\circ} \mathrm{C}$. Concentrations of pro-inflammatory cytokines IL-1 $\beta$ and IL- 6 were measured using a high-sensitive ELISA, according to the manufacturer's protocol.

RNA interference. Rat small interfering RNA (siRNA) and negative control siRNA (NC siRNA) were chemically synthesized by Shanghai GenePharma Co., Ltd. (Shanghai, 
China). Chondrocytes were seeded in 6-well plates at the density of $5.0 \times 10^{5} / \mathrm{ml}$ and then were transfected with $20 \mu \mathrm{M}$ synthesized siRNA targeting rat SIRT6. The siRNA and Lipofectamine 2000 were separately diluted in serum-free DMEM and incubated for $5 \mathrm{~min}$ at room temperature. Then the two solutions were gently mixed and incubated for $20 \mathrm{~min}$ at room temperature, prior to addition to the cells. The chondrocytes were transfected with siRNA-Lipofectamine complexes and incubated for $48 \mathrm{~h}$ at $37^{\circ} \mathrm{C}$ in a $\mathrm{CO}_{2}$ incubator, and then used in subsequent experiments.

Western blot analysis. Cellular proteins were extracted with radioimmunoprecipitation assay lysis buffer (Beyotime Institute of Biotechnology, Haimen, China) containing protease inhibitor cocktail. The sample concentration was determined by BCA kit (Beyotime Institute of Biotechnology), after that, $25 \mu \mathrm{ml}$ of samples were added to each well and electrophoresis was performed in $10 \%$ of the separation gel. Protein samples were separated by SDS-PAGE under reducing conditions and transferred to polyvinylidene difluoride membranes. The membranes were blocked with 5\% nonfat dry milk for $4 \mathrm{~h}$ at room temperature and then incubated with antibodies against rabbit anti-SIRT6 (1:1,000), anti-LC3II/I (1:1,000), anti-Beclin1 (1:1,000), anti-MCP-1 (1:400) and $\beta$-actin $(1: 1,000)$ at $4^{\circ} \mathrm{C}$ overnight. The membranes were then incubated with a goat anti-rabbit $\mathrm{IgG}$-horseradish peroxidase antibody $(1: 5,000$ or $1: 10,000)$ for $2 \mathrm{~h}$ at room temperature and subsequently washed three times with TBST buffer solution (1\% Tween-20). The relative intensity of protein bands was quantified by Quantity One Analysis Software (version 4.62; Bio-Rad Laboratories, Inc., Hercules, CA, USA) and $\beta$-actin was used as an internal control.

Reverse transcription-quantitative polymerase chain reaction $(R T-q P C R)$. Total RNA was extracted from cells using RNAiso Plus reagent. cDNAs were synthesized from total RNA using PrimeScript RT Master mix following the manufacturer's protocol. cDNA was amplified using the SYBR Premix Ex $\mathrm{Taq}^{\mathrm{TM}}$ kit. Primers are given in Table I. The relative gene expression was quantitatively analyzed by the $2^{\Delta \Delta \mathrm{Cq}}$ method (22). Data were normalized to the levels of GAPDH mRNA.

Statistical analysis. Data are presented as the mean \pm standard error from three independent experiments. Statistical significance was determined using one-way analysis of variance and the Fisher's least significant difference post hoc test, using SPSS software, version 19.0 (IBM SPSS, Armonk, NY, USA). $\mathrm{P}<0.05$ was considered to indicate a statistically significant difference.

\section{Results}

Effect of HT on cell viability in TNF- $\alpha$-induced chondrocytes. Initial experiments were performed in an anti-inflammatory cell model using HT in TNF- $\alpha$-treated chondrocytes. It is hypothesized that high concentrations of HT may exhibit cytotoxicity on chondrocytes. Cultured chondrocytes were exposed to increasing concentrations of HT $(12.5,25,50$, 100,200 and $400 \mu \mathrm{M}$ ) for $24 \mathrm{~h}$. Cell viability was examined using CCK-8 assay. As presented in Fig. 1, cell viability of
Table I. Primer sequences used for reverse transcription-quantitative polymerase chain reaction.

\begin{tabular}{lll}
\hline Gene & Primer & \multicolumn{1}{c}{ Sequence (5'-3') } \\
\hline Sirtuin 6 & Forward & 5'-GCA GTCTTCCAGTGTGGT \\
& & GT-3' \\
& Reverse & 5'-CCATGGTCCAGACTCCGT-3' \\
GAPDH & Forward & 5'-GGCACAGTCAAGGCTGAG \\
& & AATG-3' \\
& Reverse & 5'-ATGGTGGTGAAGACGCCA \\
& & GTA-3' \\
\hline
\end{tabular}

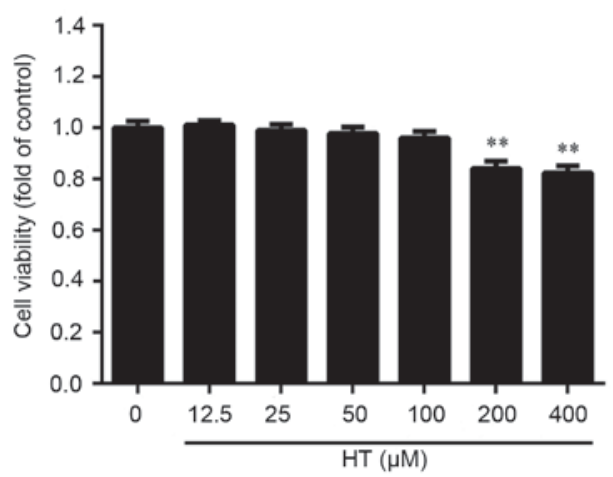

Figure 1.Effect of HT on TNF- $\alpha$-induced chondrocyte viability. Chondrocytes were incubated with different concentrations of HT $(0,12.5,25,50,100,200$ and $400 \mu \mathrm{M}$ ) for $24 \mathrm{~h}$. The cell viability was determined using a Cell Counting kit- 8 assay. The results were expressed as the mean \pm standard error $(n=3)$. ${ }^{* *} \mathrm{P}<0.01$ vs. $0 \mu \mathrm{M}$ HT. HT, hydroxytyrosol; TNF- $\alpha$, tumor necrosis factor- $\alpha$.

chondrocytes was significantly reduced following HT treatment at concentrations of 200 and $400 \mu \mathrm{M}$. At $50 \mu \mathrm{M}$ HT treatment, there was $\sim 100 \%$ cell viability and no significant cytotoxicity observed, and therefore, dosages around this concentration were used in subsequent experiments to determine the anti-inflammatory effect of HT on TNF- $\alpha$-induced cell inflammation.

HT inhibits the TNF- $\alpha$-induced inflammatory response in chondrocytes. HT has been demonstrated to exert anti-inflammatory properties, as verified by in vitro studies $(17,19)$. HT modulates inflammatory responses in murine peritoneal macrophages and human hepatoma HepG2 and Hep3B cell lines. These previous observations encouraged the authors of the present study to determine the effect of HT on inflammatory responses in TNF- $\alpha$-induced chondrocytes.

As demonstrated in Fig. 2A-C, TNF- $\alpha$ ( $5 \mathrm{ng} / \mathrm{ml})$ alone markedly increased the levels of IL- $1 \beta$, IL- 6 compared with the control, and the MCP-1 protein level was also slightly elevated compared with the control, and exposure of chondrocytes to increasing concentrations of HT (25 to $100 \mu \mathrm{M})$ resulted in a reduction in expression of these proteins, suggesting that HT exhibited an anti-inflammatory effect in a dose-dependent manner. Therefore, HT had a concentration-dependent inhibitory effect on the release levels 
A

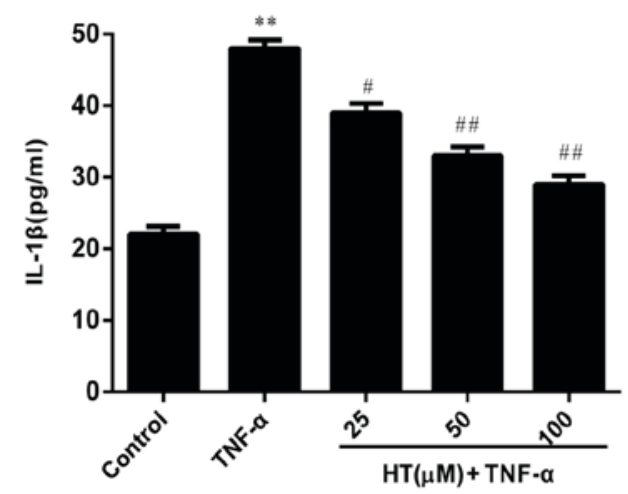

C

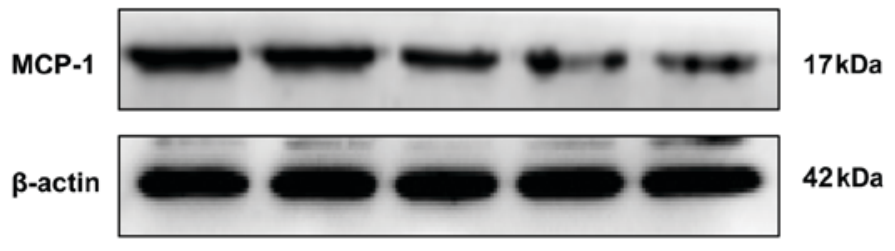

B
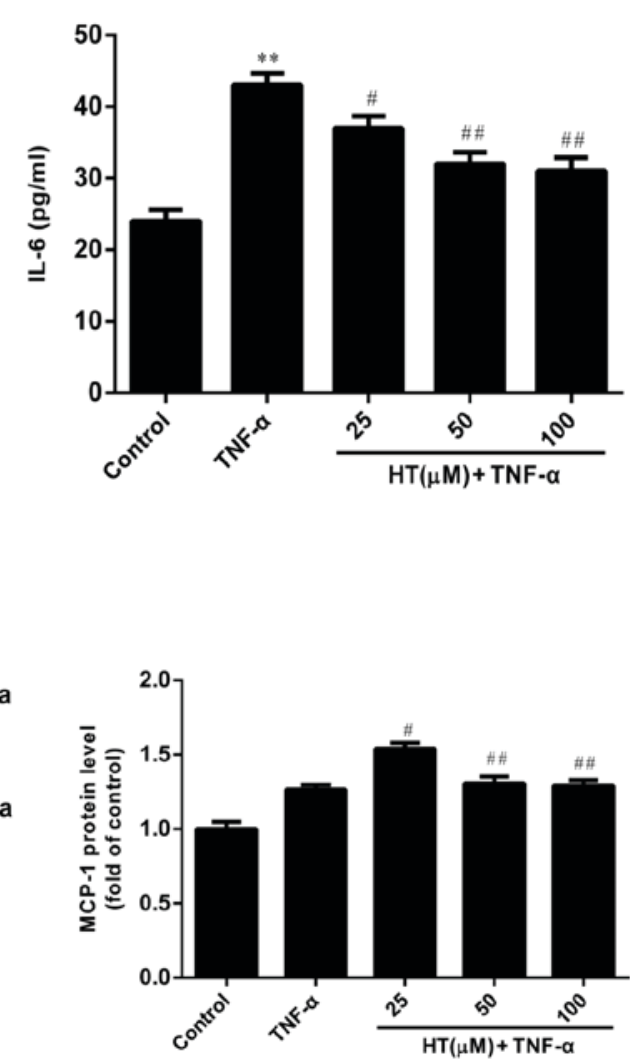

Figure 2. HT inhibits the TNF- $\alpha$-induced inflammatory response in chondrocytes. The effect of HT on the production of (A) IL-1 $\beta$ and (B) IL-6 in chondrocytes was measured by ELISA assay. Chondrocytes were pretreated with various concentrations $(25,50$ and $100 \mu \mathrm{M}) \mathrm{HT}$ for $1 \mathrm{~h}$, then stimulated with TNF- $\alpha$ $(5 \mathrm{ng} / \mathrm{ml}$ ) for $24 \mathrm{~h}$. The release of IL-1 $\beta$ and IL- 6 was measured using high-sensitive ELISA kits. (C) The expression of MCP-1 was detected by western blot analysis. The results were expressed as the mean \pm standard error $(\mathrm{n}=3) .{ }^{* *} \mathrm{P}<0.05$ vs. control group. ${ }^{~} \mathrm{P}<0.05 ;{ }^{\# \#} \mathrm{P}<0.01$ vs. TNF- $\alpha$ group. HT, hydroxytyrosol; TNF- $\alpha$, tumor necrosis factor- $\alpha$; IL-1 $\beta$, interleukin-1 $\beta$; IL-6, interleukin-6; MCP-1, monocyte chemoattractant protein 1.

of IL-1 $\beta$, IL- 6 and MCP-1 proteins in TNF- $\alpha$-stimulated chondrocytes.

HT inhibits the inflammatory response of chondrocytes via autophagy. To investigate the effect of HT on autophagy in chondrocytes, the conversion of LC3I to LC3II and the expression of the Beclin1 protein were measured using western blotting and the results were demonstrated in Fig. 3A and B. Chondrocytes treated with HT exhibited statistically significant increased expression levels of LC3-II (50 and $100 \mu \mathrm{M} \mathrm{HT}$ ) and cleaved Beclin1 $(25,50$ and $100 \mu \mathrm{M})$ compared with chondrocytes cultured with TNF- $\alpha$ alone, suggesting that HT may promote autophagy in chondrocytes under TNF- $\alpha$-induced inflammatory conditions.

Based on these findings, HT may inhibit inflammatory responses and promote autophagy in a dose-dependent manner. However, whether the anti-inflammatory effects of HT are dependent on activation of autophagy remains unclear. To determine whether the inflammatory response is mediated by autophagy induction in TNF- $\alpha$-treated chondrocytes, the effect of 3-MA, an autophagy inhibitor, on TNF- $\alpha$-induced chondrocyte inflammation was evaluated. It was demonstrated in Fig. 3C, D and E that the anti-inflammatory effect of HT was significantly blocked by pretreatment of cells with 3-MA. These results suggested that the inflammatory response of TNF- $\alpha$-induced chondrocytes was inhibited through autophagy.

HT inhibits TNF- $\alpha$-induced inflammatory response in chondrocytes through SIRT6. The aim was to determine the regulatory role of SIRT6 on the inflammatory response in chondrocytes in the presence of HT. To do this, the expression levels of SIRT6 in chondrocyte cells were determined. The protein and mRNA expression levels of SIRT6 were determined by western blot analysis (Fig. 4A) and RT-qPCR (Fig. 4B). Fig. 4A and B revealed that HT enhanced the expression of SIRT6 at the protein and mRNA level in chondrocytes compared with cells stimulated with TNF- $\alpha$ alone.

Next, it was investigated whether the anti-inflammatory effect of HT may be regulated by SIRT6 expression in chondrocytes, and it was demonstrated that the expression of SIRT6 protein and mRNA were reduced to $\sim 30 \%$ by SIRT6-specific siRNA in chondrocytes, compared with chondrocytes treated with NC siRNA (Fig. 4C and D, respectively). In support with the hypothesis that SIRT6 regulates the anti-inflammatory effect of HT in chondrocytes, siRNA knockdown of SIRT6 was associated with significantly increased release of the proinflammatory cytokines IL-1 $\beta$ and IL-6 $(\mathrm{P}<0.01$; Fig. 4E and $\mathrm{F})$. The results also revealed that knockdown of SIRT6 
A
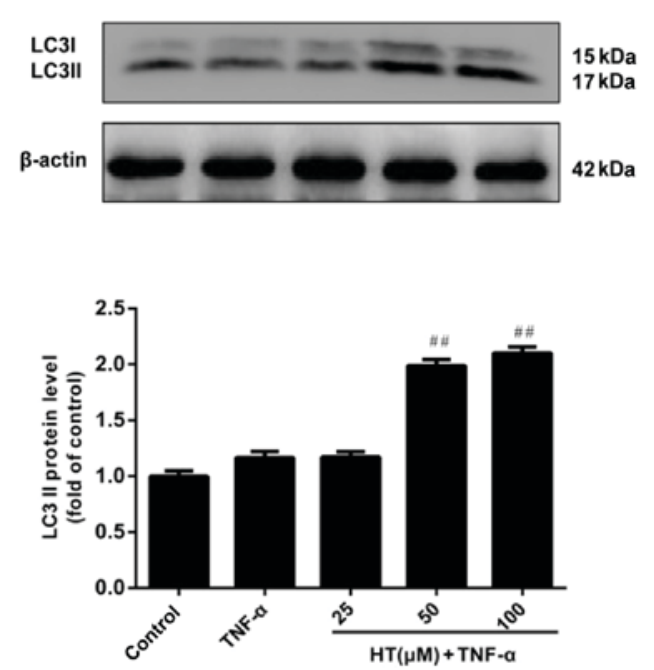

C

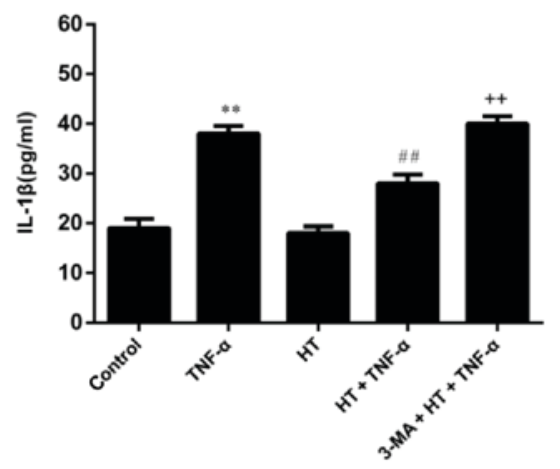

$E$

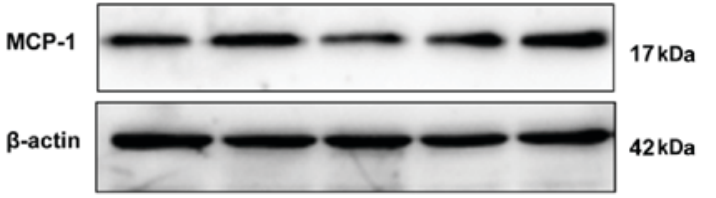

D
B

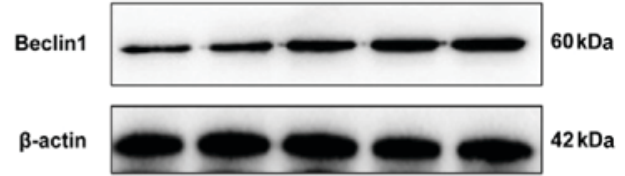

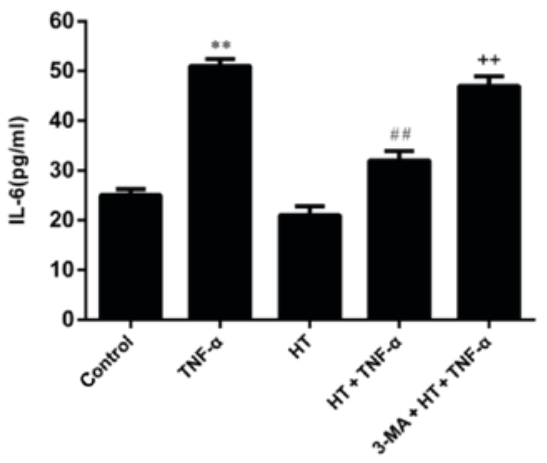

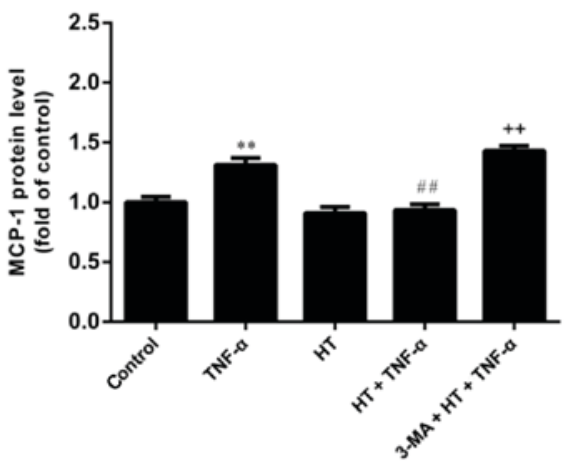

Figure 3. HT promotes autophagy in TNF- $\alpha$-induced chondrocytes. Chondrocytes were pretreated with HT at different concentrations (25, 50 and $100 \mu \mathrm{M})$ for $1 \mathrm{~h}$ and then stimulated with TNF- $\alpha(5 \mathrm{ng} / \mathrm{ml})$ for $24 \mathrm{~h}$. The protein expression of (A) LC3II and (B) Beclin1 were assessed by western blot analysis. Next, the chondrocytes were pretreated with an autophagy inhibitor, 3-MA $(5 \mathrm{mM})$, for $1 \mathrm{~h}$, and then incubated with $\mathrm{HT}(50 \mu \mathrm{M})$ for $1 \mathrm{~h}$, followed by stimulation with TNF- $\alpha$ for $24 \mathrm{~h}$. The concentration of (C) IL-1 $\beta$ and (D) IL-6 in cell supernatants were determined by ELISA. (E) The expression levels of MCP-1 were detected by western blot analysis. The results are expressed as the mean \pm standard error $(\mathrm{n}=3) .{ }^{* *} \mathrm{P}<0.01$ vs. control group. ${ }^{\# \#} \mathrm{P}<0.01 \mathrm{vs}$. TNF- $\alpha$ group. ${ }^{++} \mathrm{P}<0.01$ vs. HT+TNF- $\alpha$ group. HT, hydroxytyrosol; TNF- $\alpha$, tumor necrosis factor- $\alpha$; 3-MA, 3-methyladenine; IL-1 $\beta$, interleukin-1 $\beta$; IL-6, interleukin-6; MCP-1, monocyte chemoattractant protein 1.

increased the protein expression levels of MCP-1 compared with the NC siRNA+HT+TNF- $\alpha$ group $(\mathrm{P}<0.01$; Fig. $4 \mathrm{G})$. Taken together, these findings suggested that HT inhibited the TNF- $\alpha$-induced inflammatory response through SIRT6 activation in chondrocytes.

$H T$ inhibits $T N F$ - $\alpha$-induced inflammatory response in chondrocytes through SIRT6-mediated autophagy. The final aim of the present study was to determine whether
HT regulates autophagy through SIRT6 in TNF- $\alpha$-induced chondrocytes. To assess this, chondrocytes were transfected with SIRT6-specific siRNA to decrease SIRT6 expression and subsequently, western blotting was performed to determine the effect on LC3-1/II and Beclin1 expression levels. As presented in Fig. 5A and B, knockdown of SIRT6 reduced the levels of Beclin-1 and LC3-II in chondrocytes compared with the NC siRNA group. These results suggested that knockdown of SIRT6 significantly suppressed the expression of LC3 and 
A
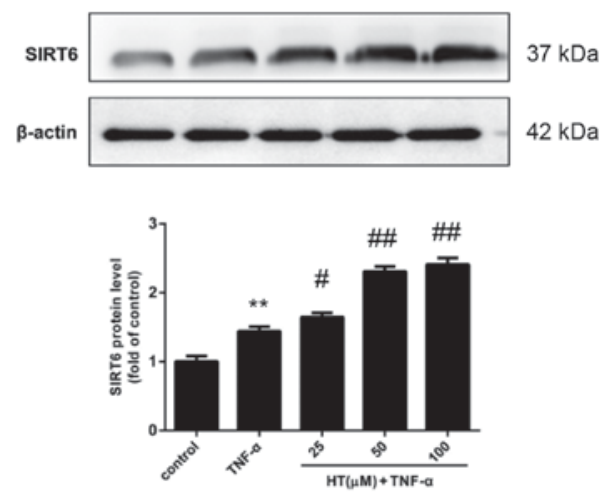

C
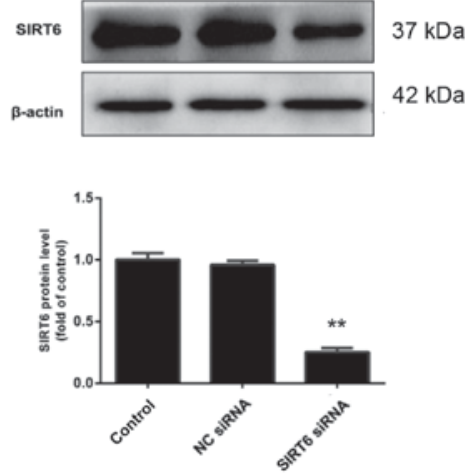

$E$

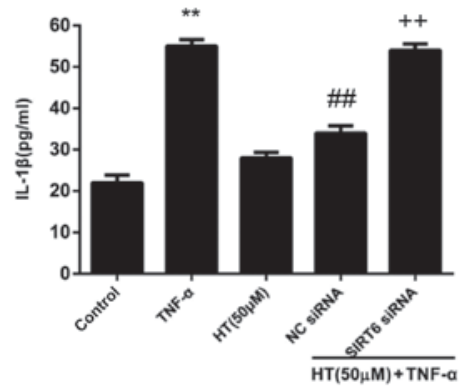

G
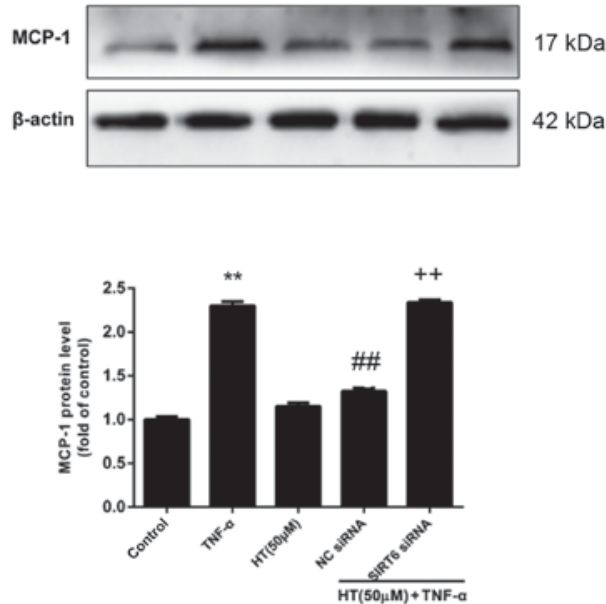

B

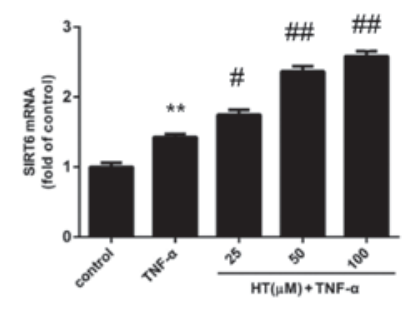

D

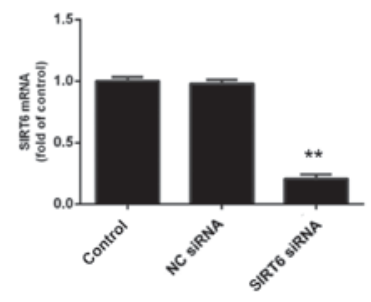

$\mathrm{F}$

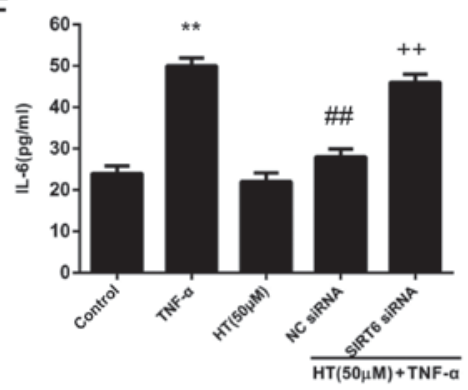

Figure 4. HT upregulates SIRT6 expression in chondrocytes stimulated with TNF- $\alpha$. Chondrocytes were pretreated with HT ( 25,50 and $100 \mu \mathrm{M})$ for $1 \mathrm{~h}$, and then stimulated with TNF- $\alpha$ for $24 \mathrm{~h}$. The expression of SIRT6 (A) protein and (B) mRNA were determined by western blot analysis and reverse transcription-quantitative polymerase chain reaction, respectively. Chondrocytes were transfected with SIRT6 siRNA or NC siRNA for $48 \mathrm{~h}$, and then the expression of SIRT6 (C) protein and (D) mRNA was assessed. The chondrocytes transfected with SIRT6 siRNA or NC siRNA were pretreated with HT (50 $\mu \mathrm{M})$ for $1 \mathrm{~h}$ and then stimulated with TNF- $\alpha(5 \mathrm{ng} / \mathrm{ml})$ for $24 \mathrm{~h}$. The release of (E) IL-1 1 and (F) IL-6 were measured using an ELISA kit. (G) The expression of MCP-1

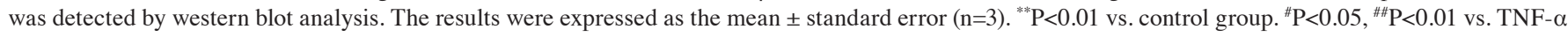
group. ${ }^{+} \mathrm{P}<0.01$ vs. NC siRNA+HT+TNF- $\alpha$ group. HT, hydroxytyrosol; TNF- $\alpha$, tumor necrosis factor- $\alpha$; SIRT6, sirtuin-6; NC, negative control. 
A
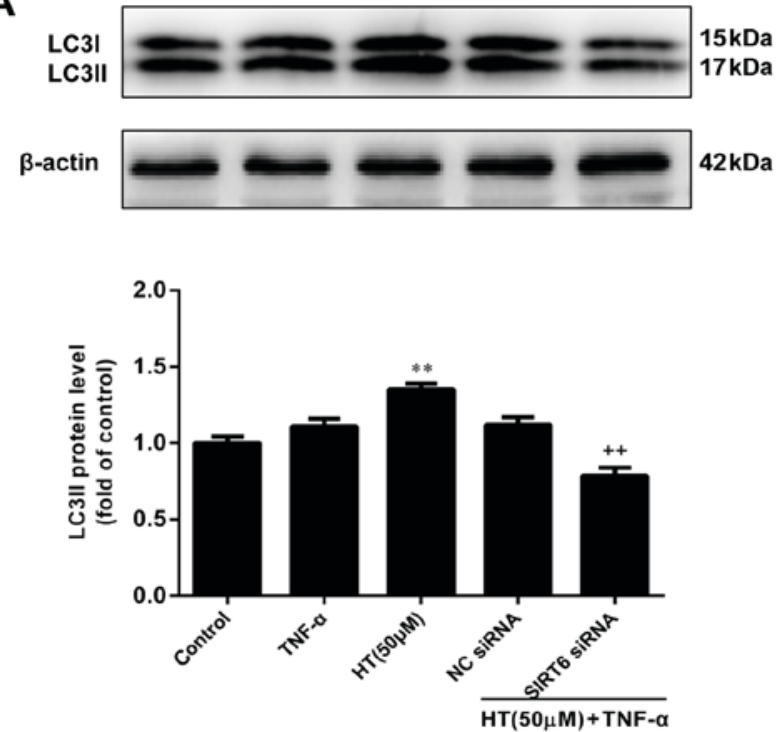

B
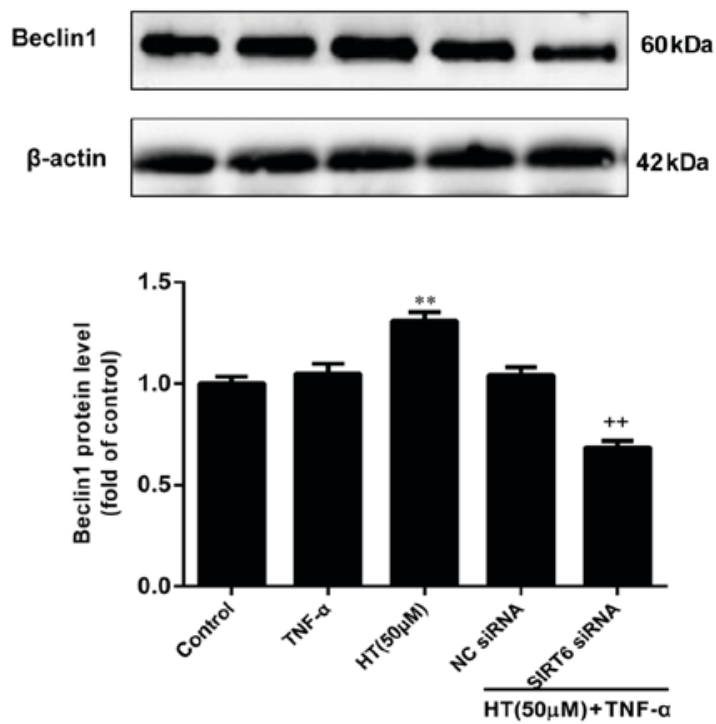

Figure 5. HT regulates autophagy through SIRT6 in TNF- $\alpha$-induced chondrocytes. The chondrocytes transfected with SIRT6 siRNA or NC siRNA were pretreated with HT $(50 \mu \mathrm{M})$ for $1 \mathrm{~h}$ and then stimulated with TNF- $\alpha(5 \mathrm{ng} / \mathrm{ml})$ for $24 \mathrm{~h}$. Expression of (A) LC3II and (B) Beclin1 were determined by western blot analysis. The results were expressed as the mean \pm standard error $(n=3) .{ }^{* *} \mathrm{P}<0.01$ vs. control group. ${ }^{++} \mathrm{P}<0.01 \mathrm{vs}$. NC siRNA $+\mathrm{HT}+\mathrm{TNF}-\alpha$ group. $\mathrm{HT}$, hydroxytyrosol; TNF- $\alpha$, tumor necrosis factor- $\alpha$; SIRT6, sirtuin-6; NC, negative control; LC3, microtubule-associated protein 1A/1B-light chain 3.

Beclin1 $(\mathrm{P}<0.01)$. Therefore, it was concluded that HT regulated the inflammatory response through SIRT6-meditated autophagy in TNF- $\alpha$-induced chondrocytes.

\section{Discussion}

Pro-inflammatory cytokines serve critical roles in the development of inflammation $(3,4)$. Chondrocytes stimulated with TNF- $\alpha$ may lead to the release of inflammatory mediators, including IL-1 $\beta$ and IL-6. These inflammatory mediators amplify inflammation and accelerate the pathophysiology of OA. Previous studies have suggested that elevated IL-1 $\beta$ in chondrocytes may lead to cartilage destruction and pain (22). Therefore, inhibition of IL- $1 \beta$ and IL- $1 \beta$-induced inflammatory mediators may possess significant clinical value in the treatment of OA. It has been reported that HT regulates the inflammatory response. In the present study, it was revealed that HT significantly inhibited IL- $1 \beta$ and IL- 6 production in TNF- $\alpha$ induced chondrocytes.

A previous study revealed that members of the Sirtuin family participate in inflammation (22). SIRT6 may suppress inflammatory responses in collagen-induced arthritis (12). Additionally, it has been revealed that SIRT6-induced autophagy is involved in oxidative stress-induced neuronal damage $(13,14)$. Although previous reports highlight SIRT6 as a negative regulator of vascular inflammation (13), the association between autophagy and the regulatory role of SIRT6 in the anti-inflammatory process has yet to be identified. Therefore, the present study hypothesized that HT may inhibit the inflammatory response of chondrocytes via SIRT6-mediated autophagy. Firstly, it was demonstrated that HT promoted autophagy in TNF- $\alpha$-induced chondrocytes. This was consistent with previous studies that revealed that HT may increase autophagy and protect chondrocytes from oxidative stress-induced DNA damage and cell death (18). In addition, the present study demonstrated that HT upregulated the expression of SIRT6 at the protein and mRNA levels in TNF- $\alpha$-stimulated chondrocytes. Knockdown of SIRT6 reduced the effect of HT on autophagy in chondrocytes by decreasing the expression levels of LC3II and Beclin1. These results suggested that HT regulates autophagy in chondrocytes through SIRT6, verifying that HT may possess effective anti-inflammatory activities in TNF- $\alpha$ induced chondrocytes and thus prevent the progression of OA.

To the best of the author's knowledge, this may be the first study to demonstrate that HT prevents TNF- $\alpha$-induced chondrocyte inflammation by enhancing SIRT6 mediated autophagy activation. The present study provided a potential mechanism associating autophagy with the onset and progression of OA $(23,24)$. SIRT6-mediated autophagy activation may be applied for the treatment of inflammatory diseases. In addition, drugs used in OA knees have failed to prevent disease progression and exhibit side effects. Local administration of glucocorticoids (GCs) has been used to treat OA. GCs inhibit local immune response through the receptor. Therefore, they serve an important role in alleviating the symptoms of patients. However, there is an amount of evidence demonstrating that GCs have a degenerative effect on certain collagen tissue, including bones, tendons and skin (25). Therefore, it is of increasing interest to identify plant-derived compounds for the treatment of OA $(26,27)$.

In conclusion, the results of the present study suggested that HT inhibits the inflammatory response of chondrocytes through SIRT6-mediated autophagy. This may provide novel insights into the association between HT, autophagy and inflammation in chondrocytes. Therefore, modulating autophagy represents an attractive future therapeutic target for treating diseases caused by inflammation. However, the present study did not confirm whether HT directly regulates SIRT6, which can affect autophagy to inhibit inflammatory response. 
Further investigation will allow better characterization of the molecular mechanisms underlying these effects. A further study will investigate which receptors or signal molecules are involved in the regulatory process of HT on SIRT6.

\section{References}

1. Roos EM and Arden NK: Strategies for the prevention of knee osteoarthritis. Nat Rev Rheumatol 12: 92-101, 2016.

2. Zhang MC, Egan B and Wang JX: Epigenetic mechanisms underlying the aberrant catabolic and anabolic activities of osteoarthritic chondrocytes. Int J Biochem Cell Biol 67: 101-109, 2015.

3. Honorati MC, Cattini L and Facchini A: IL-17, IL-1beta and TNF-alpha stimulate VEGF production by dedifferentiated chondrocytes. Osteoarthritis Cartilage 12: 683-691, 2004

4. Lianxu C, Hongti J and Changlong Y: NF-kappa Bp65-specific siRNA inhibits expression of genes of COX-2, NOS-2 and MMP-9 in rat IL-1 beta-induced and TNF-alpha-induced chondrocytes. Osteoarthritis Cartilage 14: 367-376, 2006.

5. Tetlow LC, Adlam DJ and Woolley DE: Matrix metalloproteinase and proinflammatory cytokine production by chondrocytes of human osteoarthritic cartilage: Associations with degenerative changes. Arthritis Rheum 44: 585-594, 2001.

6. Doria A, Gatto M and Punzi L: Autophagy in human health and disease. N Engl J Med 368: 1845, 2013.

7. Netea-Maier RT, Plantinga TS, van de Veerdonk FL, Smit JW and Netea MG: Modulation of inflammation by autophagy: Consequences for human disease. Autophagy 12: 245-260, 2016.

8. Chen ML, Yi L, Jin X, Liang XY, Zhou Y, Zhang T, Xie Q, Zhou X, Chang H, Fu YJ, et al: Resveratrol attenuates vascular endothelial inflammation by inducing autophagy through the cAMP signaling pathway. Autophagy 9: 2033-2045, 2013.

9. Kawahara TL, Michishita E, Adler AS, Damian M, Berber E, Lin M, McCord RA, Ongaigui KC, Boxer LD, Chang HY and Chua KF: SIRT6 links histone H3 lysine 9 deacetylation to NF-kappa B-dependent gene expression and organismal life span. Cell 136: 62-74, 2009.

10. Michishita E, Park JY, Burneskis JM, Barrett JC and Horikawa I: Evolutionarily conserved and nonconserved cellular localizations and functions of human SIRT proteins. Mol Biol Cell 16: 4623-4635, 2005.

11. Zhang J, Cheng Y, Gu J, Wang S, Zhou S, Wang Y, Tan Y, Feng W, Fu Y, Mellen N, et al: Fenofibrate increases cardiac autophagy via FGF21/SIRT1 and prevents fibrosis and inflammation in the hearts of Type 1 diabetic mice. Clin Sci (Lond) 130: 625-641, 2016.

12. Takasaka N, Araya J, Hara H, Ito S, Kobayashi K, Kurita Y, Wakui H, Yoshii Y, Yumino Y, Fujii S, et al: Autophagy Induction by SIRT6 through attenuation of insulin-like growth factor signaling is involved in the regulation of human bronchial epithelial cell senescence. J Immunol 192: 958-968, 2014.

13. Wu Y, Chen L, Wang Y, Li W, Lin Y, Yu D, Zhang L, Li F and Pan Z: Overexpression of Sirtuin 6 suppresses cellular senescence and $\mathrm{NF}-\mathrm{kB}$ mediated inflammatory responses in osteoarthritis development. Sci Rep 5: 17602, 2015.
14. Nagai K, Matsushita T, Matsuzaki T, Takayama K, Matsumoto T, Kuroda R and Kurosaka M: Depletion of SIRT6 causes cellular senescence, DNA damage, and telomere dysfunction in human chondrocytes. Osteoarthritis Cartilage 23: 1412-1420, 2015.

15. Granados-Principal S, Quiles JL, Ramirez-Tortosa CL, Sanchez-Rovira P and Ramirez-Tortosa MC: Hydroxytyrosol: From laboratory investigations to future clinical trials. Nutr Rev 68: 191-206, 2010.

16. Bulotta S, Celano M, Lepore SM, Montalcini T, Pujia A and Russo D: Beneficial effects of the olive oil phenolic components oleuropein and hydroxytyrosol: Focus on protection against cardiovascular and metabolic diseases. J Transl Med 12: 219, 2014.

17. Luo C, Li Y, Wang H, Cui Y, Feng ZH, Li H, Li Y, Wang Y, Wurtz K, Weber P, et al: Hydroxytyrosol promotes superoxide production and defects in autophagy leading to anti-proliferation and apoptosis on human prostate cancer cells. Curr Cancer Drug Targets 13: 625-639, 2013.

18. Cetrullo S, D'Adamo S, Guidotti S, Borzì RM and Flamigni F: Hydroxytyrosol prevents chondrocyte death under oxidative stress by inducing autophagy through sirtuin 1-dependent and -independent mechanisms. Biochim Biophys Acta 1860: 1181-1191, 2016

19. Gao J, Zou X, Yang L, Feng Z and Liu J: Hydroxytyrosol protects against acrolein induced preosteoblast cell toxicity: Involvement of Nrf2/Keap1 pathway. J Funct Foods 19: 28-38, 2015.

20. Aparicio-Soto M, Sánchez-Fidalgo S, González-Benjumea A, Maya I, Fernández-Bolaños JG and Alarcón-de-la-Lastra C: Naturally occurring hydroxytyrosol derivatives: Hydroxytyrosyl acetate and 3,4-dihydroxyphenylglycol modulate inflammatory response in murine peritoneal macrophages. Potential utility as new dietary supplements. J Agric Food Chem 63: 836-846, 2015.

21. Feng G, Wan Y, Balian G, Laurencin C and Li X: Adenovirus-mediated expression of growth and differentiation factor-5 promotes chondrogenesis of adipose stem cells. Growth Factors 26: 132-142, 2008.

22. Livak KJ and Schmittgen TD: Analysis of relative gene expression data using real-time quantitative PCR and the 2(-Delta Delta C(T)) method. Methods 25: 402-408, 2001.

23. Lin J, Sun B, Jiang C, Hong H and Zheng Y: Sirt2 suppresses inflammatory responses in collagen-induced arthritis. Biochem Biophys Res Commun 441: 897-903, 2013.

24. Sasaki H, Takayama K, Matsushita T, Ishida K, Kubo S, Matsumoto T, Fujita N, Oka S, Kurosaka M and Kuroda R: Autophagy modulates osteoarthritis-related gene expression in human chondrocytes. Arthritis Rheum 64: 1920-1928, 2012.

25. Shen C, Cai GQ, Peng JP and Chen XD: Autophagy protects chondrocytes fromglucocorticoids-induced apoptosis via ROS/Akt/FOXO3 signaling. Osteoarthritis Cartilage 23: 2279-2287, 2015.

26. Caramés B, Olmer M, Kiosses WB and Lotz MK: The relationship of autophagy defects to cartilage damage during joint aging in a mouse model. Arthritis Rheumatol 67: 1568-1576, 2015.

27. Liu N, Wang WB, Zhao Z, Zhang T and Song YW: Autophagy in human articular chondrocytes is cy toprotective following glucocorticoid stimulation. Mol Med Rep 9: 2166-2172, 2014. 